\title{
Índices de sustentabilidade das atividades agrícolas nos Biomas Caatinga e Mata Atlântica da Paraíba, Nordeste do Brasil
}

\author{
João Abílio Diniz ${ }^{1}$, Walter Esfrain Pereira²
}

${ }^{1}$ Instituto Federal de Rondônia - IFRO, Campus de Ariquemes. Rodovia RO-257, km 13, Zona Rural, Ariquemes-RO (CEP 76870-000). E-mail: joao.diniz@ifro.edu.br. ${ }^{2}$ Universidade Federal da Paraíba - UFPB, Campus II, Rodovia PB-079, Areia-PB (CEP 58397-000).E-mail: walterufpb@yahoo.com.br.

Resumo. Objetivando determinar e classificar índices de sustentabilidade das atividades agrícolas em 73 propriedades rurais foram pesquisadas três tipos de vegetações (Mata em Regeneração MR, Monocultivo de Pastagem - MP e Monocultivo de Milho - MM), com mais de cinco anos de cultivos sucessivos, em dois biomas (Caatinga-CA e Mata Atlântica-MA) no Estado da Paraíba, tendo como referência a aplicação de questionários quanti-qualitativos composto por diversas variáveis técnicas, econômicas e sociais. A área representativa da pesquisa compreendeu sete municípios paraibanos, sendo quatro no bioma Caatinga e três no bioma Mata Atlântica. Para classificar as propriedades rurais por índices de sustentabilidade (IS) foram consideradas variações de 0 a 1 , onde zero representavam níveis críticos e a unidade níveis excelentes, ficando estabelecidos os seguintes critérios: sustentáveis (IS $>0,80$ ); sustentabilidades ameaçadas $(0,60<$ IS $\leq 0,80)$; sustentabilidades comprometidas $(0,40<$ IS $\leq 0,60)$; insustentáveis $(0,20<$ IS $\leq 0,40)$ e seriamente insustentáveis (IS $\leq 0,20)$. Os dados foram submetidos à análise de distribuição de frequência e de análise fatorial, cujos escores foram utilizados para análise de agrupamento. Confirmou-se que aspectos técnicos, econômicos e sociais foram determinantes na classificação dos índices de sustentabilidades, mostrando que quanto melhores maiores foram os IS. Das 73 propriedades avaliadas, 34 ou 46,58\% foram diagnosticadas como insustentáveis, sendo 19 $(55,88 \%)$ na Mata Atlântica e 15 (44,12\%) na Caatinga. Por outro lado, 39 ou 53,42\% foram classificadas em condições de serem administradas, podendo com adoções de medidas racionais tornaremse sustentáveis. Independentemente das condições analisadas verificou-se ser oportuno e racional definir o uso do solo de acordo com as potencialidades e limitações das atividades agrícolas dos diferentes biomas do Estado da Paraíba. Portanto, para alcançar o nível desejável das propriedades rurais (IS $>0,80$ ), propõem-se um conjunto de ações racionais fundamentadas em avanços nos aspectos técnicos, econômicos, sociais e ambientais, tais como: melhoramento no grau de instrução dos proprietários, adoção de práticas conservacionistas, conscientização no sentido de promover a defesa da sustentabilidade, estímulo às experiências com agricultura sustentável, observância dos cuidados necessários antes, durante e após aplicações de defensivos, incentivo a destinação de embalagens
Recebido: 15/12/2015

Aceito: 28/12/2015

Publicado: 31/12/2015

Acesso Aberto

Artigo completo

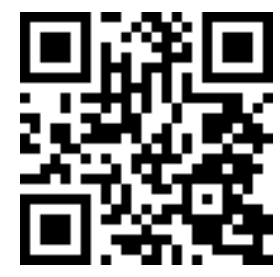


vazias aos locais apropriados, encoivaramento e decomposição da vegetação desmatada, uso de equipamento de proteção individual durante aplicação de defensivos agrícolas, armazenamento dos insumos agrícolas em locais reservados, coleta adequada do lixo domiciliar e destinação do esgoto sanitário em rede coletora.

Palavras-chave: Propriedade rural, Monocultivo, Vegetação.

Abstract. Sustainability indices of agricultural activities in biomes Caatinga and Atlantic Forest Paraíba, Northeast Brazil. Aiming to determine and classify sustainability indices of agricultural activities on 73 farms were surveyed three types of vegetation (Forest in Regeneration - MR, Pasture Monocropping - MP and Corn Monocropping - MM) with over five years of successive growth in two biomes (CA-Catting and Atlantic Forest-MA) in the State of Paraiba, with reference to the application of quantitative and qualitative questionnaires composed of several technical variables, economic and social. The representative of the survey area comprised seven cities in Paraíba, four in the biome Catting and three in the Atlantic Forest. To classify farms for Sustainability Indices (IS) variations were considered $0-1$, where zero represented critical levels and drive excellent levels, the following criteria being established: sustainable (IS > 0.80); threatened sustainabilities $(0.60<$ IS $\leq 0.80$ ); committed sustainabilities $\quad(0.40<$ IS $\leq 0.60)$; unsustainable $(0.20<$ IS $\leq 0.40)$ and seriously unsustainable (IS $\leq 0.20)$. Data were submitted to frequency distribution analysis and factor analysis, whose scores were used for cluster analysis. It was confirmed that technical, economic and social aspects were decisive in the classification of sustainabilities indices, showing that the best were the largest IS. Of the 73 properties assessed, 34 or $46.58 \%$ were diagnosed as unsustainable, 19 (55.88\%) in the Atlantic Forest and 15 (44.12\%) in the Catting. Moreover, 53.42 or $39 \%$ were classified into conditions to be administered may adoptions rational measures to be sustainable. Regardless of the examined conditions was found to be timely and rational define land use in accordance with the potential and limitations of the agricultural activities of the different biomes of the State of Paraíba. Therefore, to achieve the desired level of farms (IS > 0.80), propose a set of rational actions based on advances in technical, economic, social and environmental benefits, such as improving the educational level of the owners, adoption of conservation practices, awareness to promote the defense of sustainability, stimulating experiences with sustainable agriculture, observance of care necessary before, during and after pesticide applications, encouraging the allocation of empty containers to the appropriate local, encoivarament and decaying vegetation deforested, personal protective equipment use during application of pesticides, storage of agricultural inputs in reserved places, proper collection and disposal of household waste in the sewage collection network.

Keywords: Rural property, Monoculture, Vegetation. 


\section{Introdução}

Aspectos técnicos, econômicos e sociais podem fornecer indicativos importantes sobre a sustentabilidade do solo merecendo, portanto, atenção especial no meio científico. Analisando-se conjuntamente estes aspectos com as características edafo-climáticas e vegetativas dos biomas torna-se possível ter uma visão mais ampla dos sistemas de manejos com reflexo direto no grau de sustentabilidade das atividades agrícolas nas propriedades rurais.

Embora tenha havido avanços significativos no entendimento do tema sustentabilidade, ainda se fazem necessárias outras descobertas no que diz respeito às inter-relações da natureza (Canuto, 2003). Assim, torna-se oportuno estudar os Índices de Sustentabilidade (IS) com base nas principais atividades agrícolas nos vários biomas existentes. Desenvolver uma pesquisa desta envergadura envolvendo mais de dois biomas, além de onerosa e demorada requer desproporcional esforço físico e mental, razão pela qual esta se restringiu inicialmente apenas aos domínios vegetativos do Estado da Paraíba.

O grande desafio na quantificação ou qualificação de trabalhos nesta linha de pesquisa reside na confiabilidade das metodologias utilizadas neste sentido (Masera et al., 1999). Não obstante, autores como Melo (1999), Souza et al. (2001), Branco (2003) e Lopes et al. (2009) respaldaram a ideia de que isto seja possível estabelecendo-se índices de sustentabilidades através de escores atribuídos as variáveis aplicadas em questionários apropriados. Da mesma forma que se aceita na ciência da saúde humana pesquisas relacionando peso e altura ideal, teor de gordura ao nível de colesterol, batimentos cardíacos à pressão arterial e outros, nada mais natural do que reconhecer que as atividades agrícolas podem ser classificadas por índices que indicam se a propriedade rural encontra-se sustentável, ameaçada, comprometida, insustentável ou seriamente insustentável.

Mesmo admitindo-se a dificuldade do estabelecimento desses indicadores em função das multivariações da sustentabilidade ambiental (Toledo e Nicolella, 2002), isto se torna possível através da síntese de informações, priorizando apenas o essencial do analisado (Palácio, 2004), especialmente se empregar a estatística multivariada com análise fatorial/análise de componentes principais (Carneiro Neto et al., 2008; Palácio, 2004).

Portanto, a sustentabilidade das atividades agrícolas nas propriedades rurais, tão dificultosa de ser entendida através das metodologias tradicionais devido as suas inúmeras peculiaridades e questionamentos no meio científico (Masera et al., 1999; Lopes et al., 2009), pode ter maior amparo e respaldo na literatura quando os dados forem submetidos a análise de distribuição de frequência e de análise fatorial, utilizando-se escores para análise de agrupamento, conforme proposto nesta pesquisa.

Este trabalho teve como objetivo determinar e classificar índices de sustentabilidade em 73 propriedades rurais com três atividades agrícolas comuns (Mata em Regeneração-MR, Monocultivo de Pastagem-MP e Monocultivo de MilhoMM) em dois Biomas (Caatinga-CA e Mata Atlântica-MA) no Estado da Paraíba, tendo como referência a aplicação de questionários quanti-qualitativos composto por diversas variáveis técnicas, econômicas e sociais.

\section{Material e métodos}

\section{Caracterização do experimento}

A área pesquisada compreendeu sete municípios do Estado da Paraíba, região Nordeste do Brasil, sendo quatro na Caatinga e três na Mata Atlântica (Figura 1).

Todas as propriedades foram georeferenciadas pelo Google Earth (2014). As coordenadas geográficas foram definidas na entrada da propriedade rural (Figuras 2 e 3).

Este trabalho foi do tipo "estudo de caso" (Andrade, 1991), tendo-se buscado ilustrar generalizações que mesmo pouco abrangentes possibilitassem extrapolações em diferentes contextos e situações. 
Inicialmente, foram aplicados questionários simplificados de campo (Tabela 1), buscando que o respondente compreendesse com clareza o que fosse perguntado (Bello, 2008). Para determinação do tamanho da amostra foi

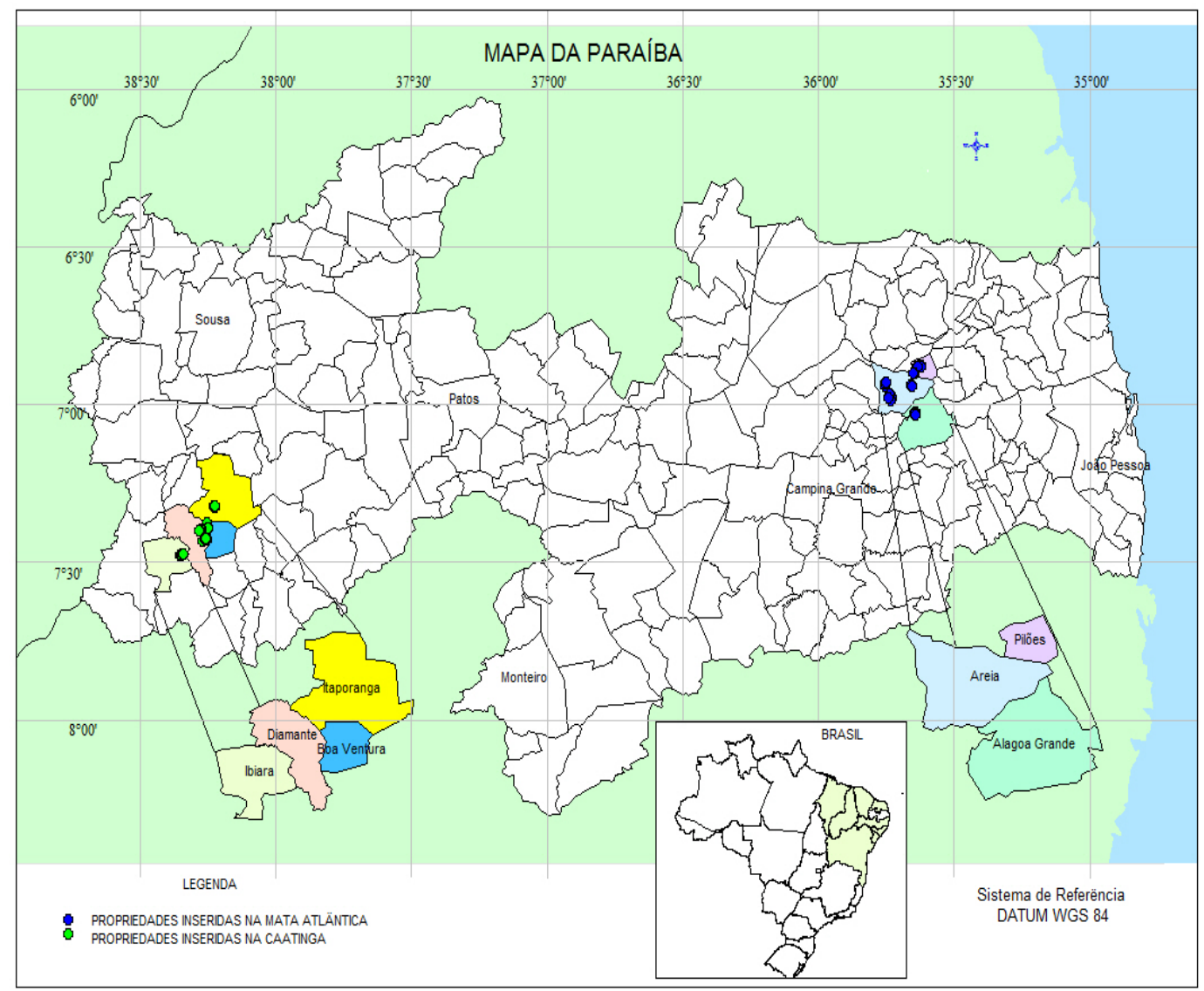

Figura 1. Localização dos municípios e áreas amostradas nos dois biomas da Paraíba. 


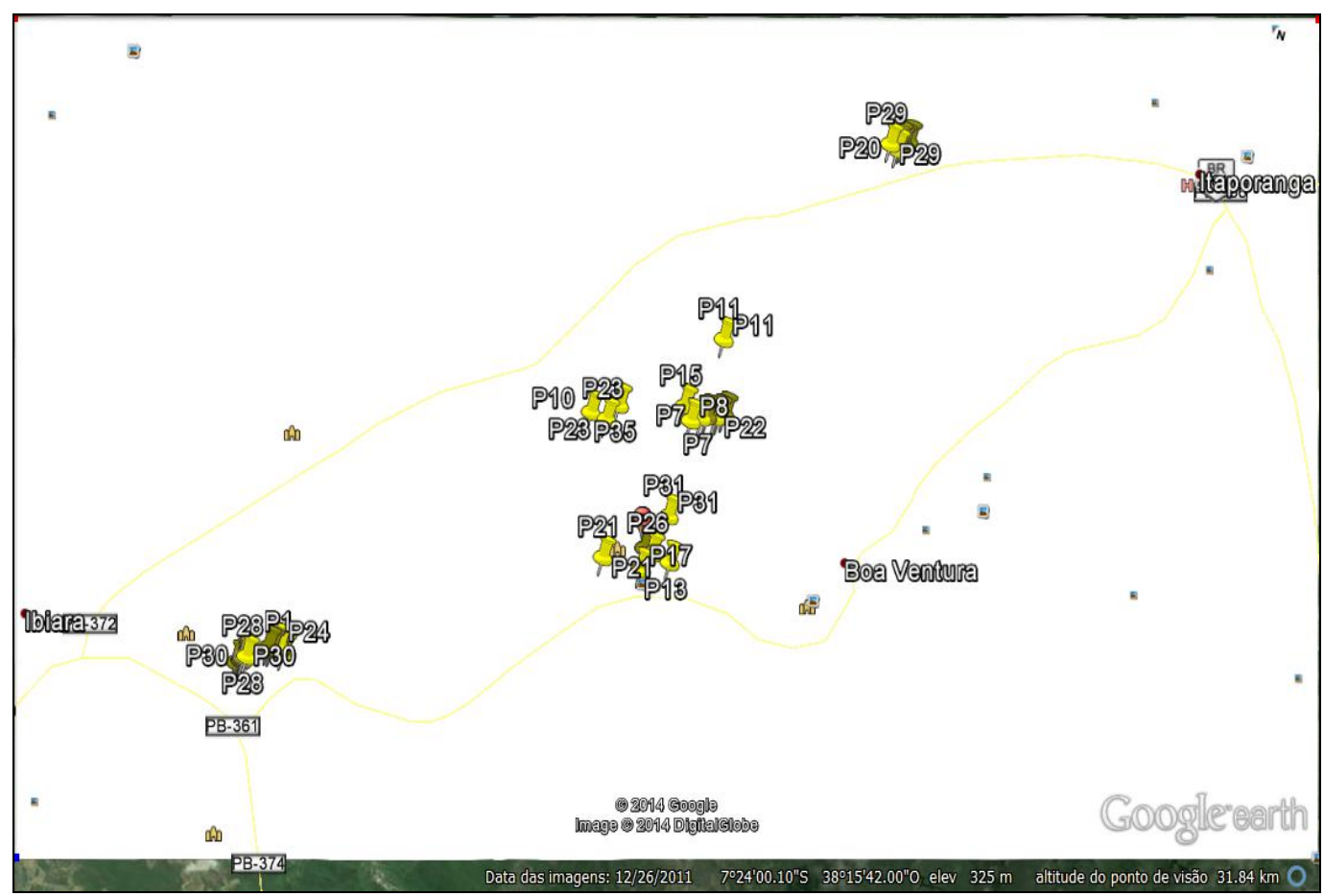

Figura 2. Aplicações de questionários no bioma Caatinga por municípios e respectivas propriedades rurais georeferenciadas (Google Earth, 2014).

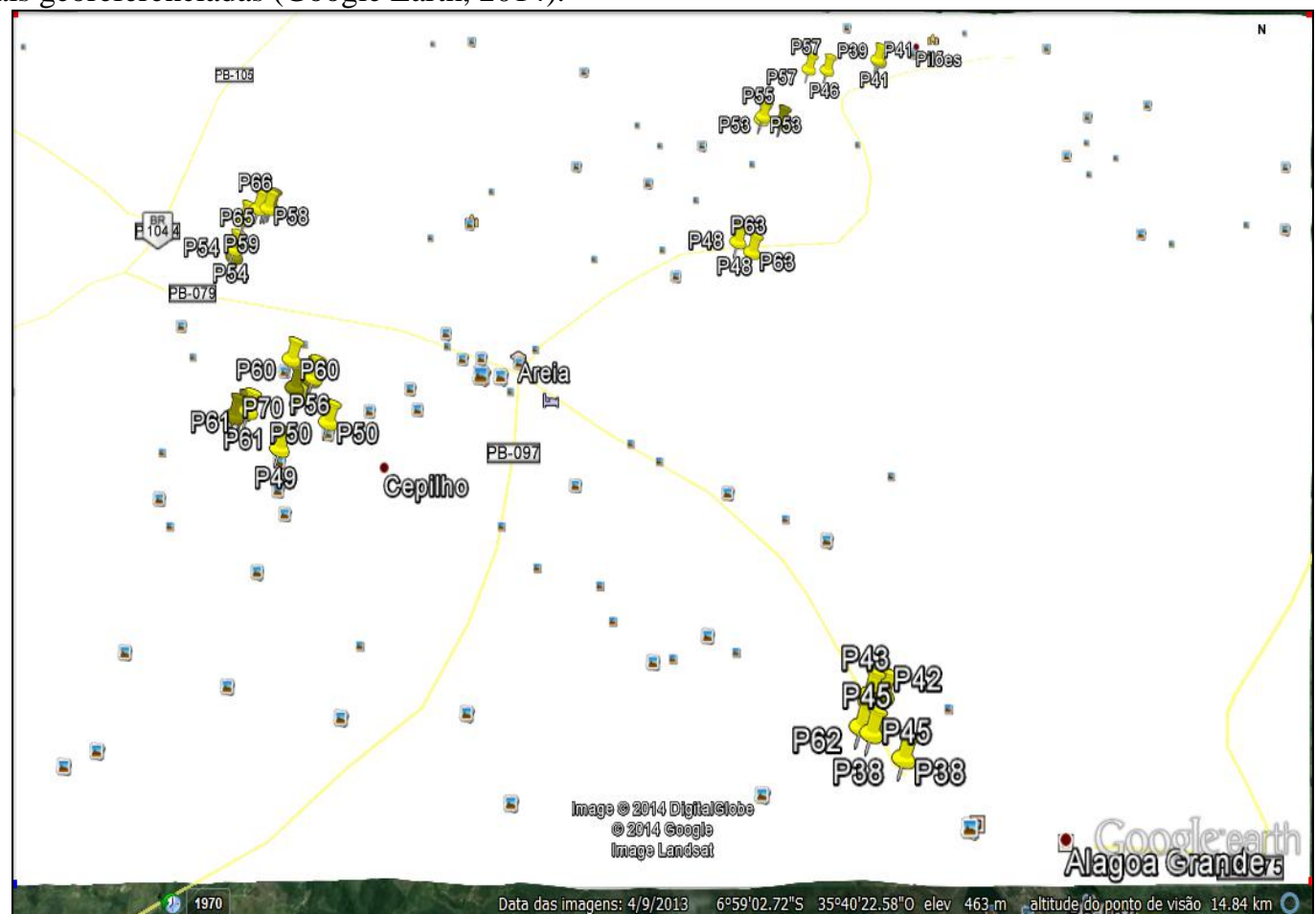

Figura 3. Aplicações de questionários no bioma Mata Atlântica por municípios e respectivas propriedades rurais georeferenciadas (Google Earth, 2014).

Tabela 1. Características técnicas, econômicas e sociais dos proprietários entrevistados nos dois biomas (Caatinga e Mata Atlântica).

\begin{tabular}{lllll}
\hline Variáveis & Respostas & Escore (I) & Frequência & Percentual \\
\hline
\end{tabular}




\begin{tabular}{lcccc}
\hline Qual seu grau de & Analfabeto & 0 & 7 & 9,59 \\
instrução? & $1^{\circ}$ Grau incompleto & 0,15 & 38 & 52,05 \\
& $1^{\circ}$ Grau completo & 0,30 & 8 & 10,96 \\
& $2^{\circ}$ Grau incompleto & 0,45 & 5 & 6,85 \\
& $2^{\circ}$ Grau completo & 0,60 & 4 & 5,48 \\
& Superior incompleto & 0,80 & 1 & 1,37 \\
& Superior completo & 1 & 10 & 13,70 \\
Quais práticas de & Plantio direto & 0,20 & 0 & 0 \\
conservação usam & Rotação de cultura & 0,20 & 0 & 0 \\
na sua & Cobertura morta & 0,20 & 0 & 0 \\
propriedade? & Quebra-vento & 0,20 & 0 & 100 \\
Qual sua opinião & Outra & 0,20 & 73 & 28,77 \\
sobre & Boa & 1 & 21 & 63,01 \\
sustentabilidade? & Regular & 0,50 & 46 & 8,22 \\
Tem experiência & Ruim & 0 & 6 & 36,99 \\
com agricultura & Sim & 1 & 27 & 63,01 \\
sustentável? & Não & 0 & 46 & 36,99 \\
Quais os cuidados & Toma banho & 0,50 & 6,85 \\
após aplicação de & Lava as roupas & 0,30 & 27 & 0 \\
defensivos? & Lava as mãos & 0,20 & 5 & 0,16 \\
\hline
\end{tabular}


Tabela 1. Continuação.

\begin{tabular}{|c|c|c|c|c|}
\hline Variáveis & Respostas & Escore (I) & Frequência & Percentual \\
\hline \multirow{5}{*}{$\begin{array}{l}\text { O que faz com as } \\
\text { embalagens } \\
\text { vazias } \\
\text { defensivos? }\end{array}$} & Devolve ao fornecedor & 1 & 0 & 0 \\
\hline & Joga no lixão & 0,40 & 8 & 10,96 \\
\hline & Deixa no terreno, depois enterra & 0,10 & 35 & 47,95 \\
\hline & Reutiliza & 0 & 0 & 0 \\
\hline & Outros (queima etc) & 0,30 & 30 & 41,10 \\
\hline \multirow{3}{*}{$\begin{array}{l}\text { O que faz com a } \\
\text { vegetação } \\
\text { desmatada? }\end{array}$} & Coivaras e deixa em & 1 & 2 & 2,74 \\
\hline & decomposição & 0,5 & 71 & 97,26 \\
\hline & $\begin{array}{l}\text { Coivaras e queima } \\
\text { Queima em toda área }\end{array}$ & 0 & 0 & 0 \\
\hline \multirow{6}{*}{$\begin{array}{ll}\text { Usa proteção na } \\
\text { aplicação } & \text { dos } \\
\text { defensivos? } & \end{array}$} & Máscara & 0,40 & 8 & 10,96 \\
\hline & Luvas & 0,20 & 6 & 8,22 \\
\hline & Óculos & 0,20 & 8 & 10,96 \\
\hline & Botas & 0,10 & 58 & 79,45 \\
\hline & Avental & 0,10 & 0 & 0 \\
\hline & Não usa proteção & 0 & 1 & 1,37 \\
\hline \multirow{2}{*}{$\begin{array}{l}\text { Como faz o } \\
\text { armazenamento } \\
\text { dos adubos? }\end{array}$} & Em local reservado & 1 & 51 & 69,86 \\
\hline & Em casa & 0 & 22 & 30,14 \\
\hline Coeficiente de & Excelente & 1 & 0 & 0 \\
\hline Uniformidade de & Bom & 0,70 & 73 & 100 \\
\hline Distribuição $\quad-$ & Regular & 0,40 & 0 & 0 \\
\hline CUD? & Ruim & 0 & 0 & 0 \\
\hline \multirow{4}{*}{$\begin{array}{l}\text { Qual o destino do } \\
\text { lixo domiciliar? }\end{array}$} & Coletado & 1 & 0 & 0 \\
\hline & Queimado & 0,30 & 25 & 34,25 \\
\hline & Enterrado & 0,40 & 47 & 64,38 \\
\hline & Céu aberto & 0 & 1 & 1,37 \\
\hline \multirow{3}{*}{$\begin{array}{l}\text { Qual o destino do } \\
\text { esgoto sanitário? }\end{array}$} & Rede coletora & 1 & 0 & 0 \\
\hline & Fossa séptica & 0,70 & 73 & 100 \\
\hline & Não há & 0 & 0 & 0 \\
\hline
\end{tabular}

Adaptado: Lopes et al. (2009).

empregada a técnica probabilística sugerida por Fonseca e Martins (1996), fundamentando-se na seguinte equação:

$$
n=\frac{z^{2} p q N}{d^{2}(\mathrm{~N}-1)+z^{2} p q}
$$

Foram considerados todos os elementos componentes da população podendo compor a amostragem, com probabilidade igual e diferente de zero. Considerou-se também com base em dados municipais disponíveis, que nos sete municípios pesquisados tinham aproximadamente $\quad 7.560$ propriedades agrícolas e que deste total 10\% (756 propriedades) possuiam os três tipos de vegetações desejáveis, ou seja, mata, pastagem e milho em sistemas de monocultivos com mais de cinco anos de atividades.

Considerou-se ainda, conforme preconizado por Lopes et al. (2009), que o erro amostral (d) deveria ser de $10 \%$ de probabilidade, com desvio padrão $(Z)$ de 1,645 em um universo de 756 propriedades com potencialidades de serem pesquisadas (N) e com percentuais dos elementos de amostras favoráveis (p) e desfavoráveis (q) de $50 \%$ para cada um, determinando-se assim o tamanho da amostra pela equação mencionada.

Para efeito de maior confiabilidade e margem de segurança à pesquisa, foram aumentadas 10 propriedades no tamanho da amostra, passando de 63 para 73 propriedades avaliadas. 
Para definir os IS das propriedades rurais, foi adotada a metodologia de Vasconcelos e Torres Filho (1994), aceita por outros pesquisadores (Melo, 1999; Carneiro Neto et al, 2008) que consideraram em suas pesquisas variações de 0 a 1 nos índices, dividindo-se as unidades produtivas nas seguintes categorias:

- Sustentável: IS > 0,80;

- Sustentabilidade Ameaçada: 0,60

$<$ IS $\leq 0,80$;

- Sustentabilidade Comprometida:

$0,40<$ IS $\leq 0,60$;

- Insustentável: 0,20 < IS $\leq$ 0,40;

- Seriamente Insustentável: IS < 0,20 .

O IS da propriedade rural foi definido pelo somatório do produto entre o escore de cada variável (I), descrito por Barreto et al. (2005) e Carneiro Neto et al. (2008), e o termo de ponderação dos indicadores no índice (p), utilizando-se a expressão algébrica, a saber:

$$
\mathrm{IS}=\mathrm{p}_{1} \cdot \mathrm{I}_{1}+\mathrm{p}_{2} \cdot \mathrm{I}_{2}+\ldots+\mathrm{p}_{\mathrm{i}} \cdot \mathrm{I}_{\mathrm{i}}
$$

Para determinação do $\mathrm{p}_{\mathrm{i}}$ foi utilizada a equação sugerida por Palácio (2004), ou seja:

$p i=\frac{\left(F_{1} \cdot C_{1}\right)+\left(F_{2} \cdot C_{2}\right)+\cdots+\left(F_{i} \cdot C_{i}\right)}{\left(\begin{array}{llll}F_{1} \cdot \sum_{1}^{n} & C 1)+\left(F_{2} \cdot \sum_{1}^{n}\right. & \left.C_{2}\right)+\cdots+\left(F_{i} \cdot \sum_{1}^{n}\right. & C_{i}\end{array}\right)}$

De acordo com a fórmula $\mathrm{p}_{\mathrm{i}}$ foi $\mathrm{o}$ peso associado a cada parâmetro de sustentabilidade; $F_{i}$ o autovalor de cada fator; $C_{i}$ a explicabilidade da variável em relação a componente principal.

\section{Aplicações de questionários sobre sustentabilidade ambiental}

Os questionários foram aplicados nas propriedades rurais ouvindo-se os membros de cada família, utilizando-se formulário padrão contendo 12 perguntas com possíveis respostas e respectivos valores, seguindo adaptações da metodologia de Lopes et al. (2009) (Tabela $1)$.
Para as respostas fornecidas foram atribuídos valores conforme adaptações de questionários aplicados em pesquisas semelhantes realizadas por Lopes et al. (2009), seguindo o modelo definido na Tabela 1.

\section{Análise estatística}

Os dados foram submetidos a análise de distribuição de frequência e a análise fatorial, cujos escores foram utilizados para análise de agrupamento. O software utilizado foi o SAS 9.3 (SAS, 2011).

\section{Resultados e discussão}

\section{sociais}

\section{Condições técnicas, econômicas e}

As condições técnicas, econômicas e sociais foram avaliadas com base em questionários aplicados em campo, considerando-se propriedades rurais com três tipos de explorações agrícolas em comum em dois biomas diferentes (Tabela 1).

Em geral, estes fatores influenciaram nos índices de sustentabilidades alcançados, verificando-se uma tendência, salvo raríssimas exceções que só confirmaram a regra, que aquelas propriedades cujos participantes tiveram essas condições favorecidas obtiveram índices melhores, ao passo que as outras desfavorecidas nestes aspectos tiveram índices piores.

Tendências semelhantes também foram registradas por Lopes et al. (2009), estudando os referidos fatores em região de perímetro irrigado no Estado do Ceará, confirmando que as propriedades melhores avaliadas em índices de sustentabilidade, via de regra, apresentaram de fato estas condições mais favorecidas. Outros autores (Melo, 1999; Souza et al., 2001; Branco, 2003), apontaram de forma análoga a existência da inter-relação da sustentabilidade com fatores dessa natureza.

Dos 73 proprietários pesquisados, 38 responderam ter primeiro grau incompleto e 7 serem analfabetos, o que foi 
considerado um fator preocupante, por isto representar maior dificuldade em absolver conhecimentos técnicos e, consequentemente, ter uma vida melhor econômico-socialmente.

Embora todos tenham respondido adotar algum tipo de prática conservacionista na propriedade, a grande maioria, ou seja, 46 indagados sobre a opinião que tinham sobre sustentabilidade disseram ser regular e apenas 21 responderam ser boa, tendo 6 entrevistados péssima aceitação sobre o tema, refletindo esta última opinião negativamente sobre os índices de sustentabilidade (IS).

Dentre as práticas conservacionistas utilizadas, o destaque ficou por conta da adoção do pousio no monocultivo do milho, colocando as 73 propriedades em situações semelhantes neste quesito, o que acabou esta pergunta não sendo um fator diferencial nas comparações e avaliações realizadas. Realmente, Masera et al (1999) alertaram sobre as dificuldades a serem enfrentadas nas metodologias utilizadas na quantificação e qualificação da sustentabilidade em pesquisas científicas, sendo certamente estes tipos de respostas obtidas, ou seja, iguais para todos uma destas.

Sobre a pergunta relacionada à experiência com agricultura sustentável 46 responderam não e apenas $27 \mathrm{sim}$; já em relação à pergunta sobre os cuidados após a aplicação dos defensivos, 41 afirmaram lavar as mãos, 5 lavar as roupas e 27 tomar banho.

A pergunta sobre a devolução das embalagens vazias foi respondida por 35 dizendo deixar no terreno e depois enterrar, por 8 afirmando jogar no lixão e por 30 afirmando que fizeram outros usos com tais recipientes ou queimaram.

Quanto ao destino da vegetação desmatada, 71 disseram encoivarar e queimar e apenas 2 afirmaram encoivarar e deixar em decomposição; já sobre o uso de equipamento de proteção individual, 58 confirmaram usar botas, 6 usar luvas, 8 usar máscara e apenas 1 não usar proteção nenhuma.
$\mathrm{O}$ armazenamento dos insumos foi respondido por 22 como sendo em casa e por 51 como sendo em local reservado.

$O$ destino do lixo domiciliar foi respondido por 47 como sendo enterrado, por 25 como queimado e por apenas 1 como deixado a céu aberto; enquanto que em relação ao destino do esgoto sanitário, todos os 73 afirmaram fluírem em direção as fossas sépticas, razão também não ter esta pergunta assim como a referente às práticas conservacionistas apresentado neste particular diferenças relevantes nas comparações e avaliações realizadas. Neste último caso, prevaleceram aquelas dificuldades mencionadas por Masera et al. (1999) em relação a adequação e aceitação das metodologias utilizadas nas mensurações dos índices de sustentabilidades.

No geral observou-se que das respostas fornecidas, muitas se relacionaram aos fatores comprometedores da sustentabilidade ambiental, o que também foi verificado por Lopes et al. (2009). Estas constatações induziram ao pensamento de que apesar da aparente evolução técnico-econômico-social no país, muito ainda precisa ser feito no sentido de melhorar nestes aspectos.

Dentre estas ações em benefício da sustentabilidade e da humanidade, as interpretações dos dados obtidos sugeriram que deve ocorrer na zona rural maior esforço governamental e não governamental para minimizar o problema da falta de alfabetização, promovendo conscientização dos agricultores da importância do avanço no grau de instrução não só na condição de cidadão, mas também na proteção ambiental. Embora tenham sido encontrados indivíduos com grau de instrução superior praticando atos de degradações nos usos dos recursos naturais, presumiu-se que via de regra estes tenham tido, pelo melhor entendimento das questões ambientais, mais consciência do que deve ser feito em benefício dos agroecossistemas, bem como das penalidades que possam sofrer caso não sigam as determinações da lei. $\mathrm{Na}$ Alemanha, cujo grau de instrução na zona 
rural supera sobremaneira o do Brasil, praticamente não existindo analfabetismo, leis ambientais estabelecerem diretrizes penalizando àqueles que contrariarem 0 manejo correto do solo (Horn e Herner, 2008), o que poderia também acontecer aqui se o nível educacional estivesse num patamar melhor.

Índices de sustentabilidades das atividades agrícolas

Foram identificadas e

caracterizadas 37 propriedades rurais na Caatinga e 36 na Mata Atlântica que preenchessem os requisitos desejados, ou seja, tivessem como vegetações comuns matas em regenerações, pastagens e milharais com mais de cinco anos de uso em forma de monocultivos (Figuras 4 e 5).

Analisando separadamente os biomas verificou-se que na Caatinga as 10 melhores propriedades em termos de IS foram P11, P15, P8, P10, P34, P29, P26, $\mathrm{P} 35$, P21 e P17; enquanto que as piores foram P19, P24, P9, P14, P16, P25, P27, P31, P4, P2 e P22. Já no bioma Mata Atlântica, as melhores foram P41, P38, P42, P56, P60, P45, P71, P50, P40 e P39, sendo P72, P65, P61, P62, P58, P55, P44, P46, P64 e P63 as piores (Tabela 2).

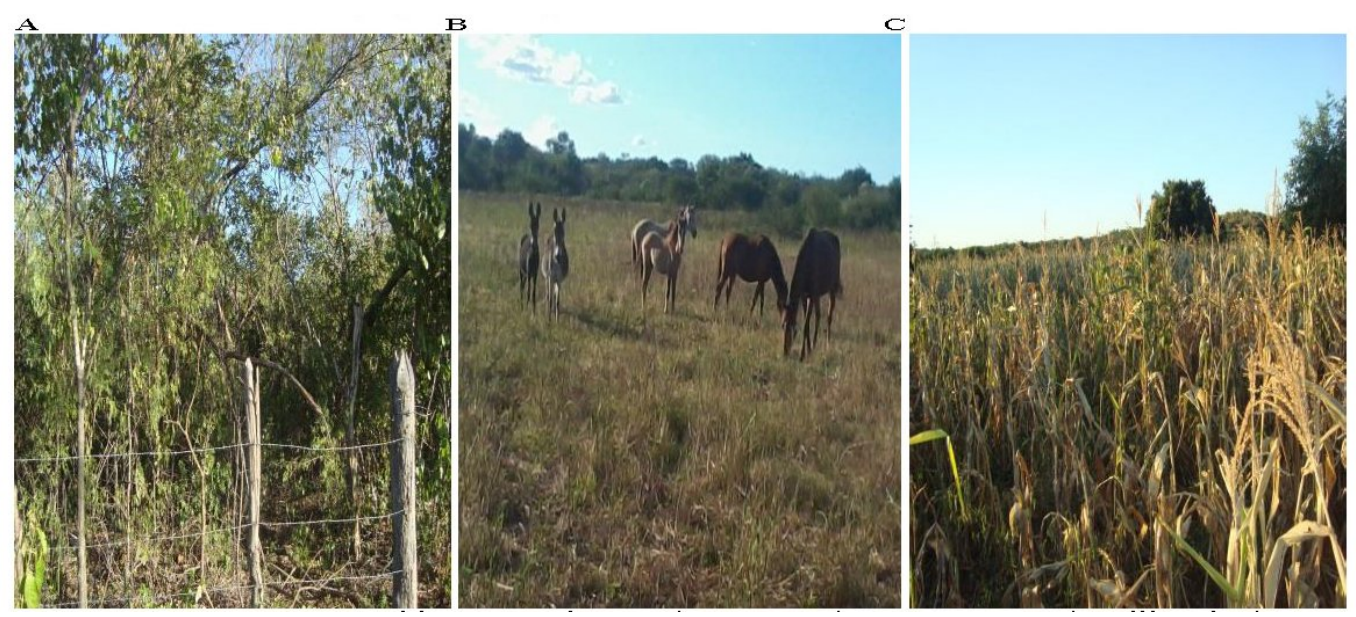

Figura 4. Vegetações no bioma Caatinga: A) Matas, B) Pastagens e C) Milharais (Fotos: Marcello, 2012).

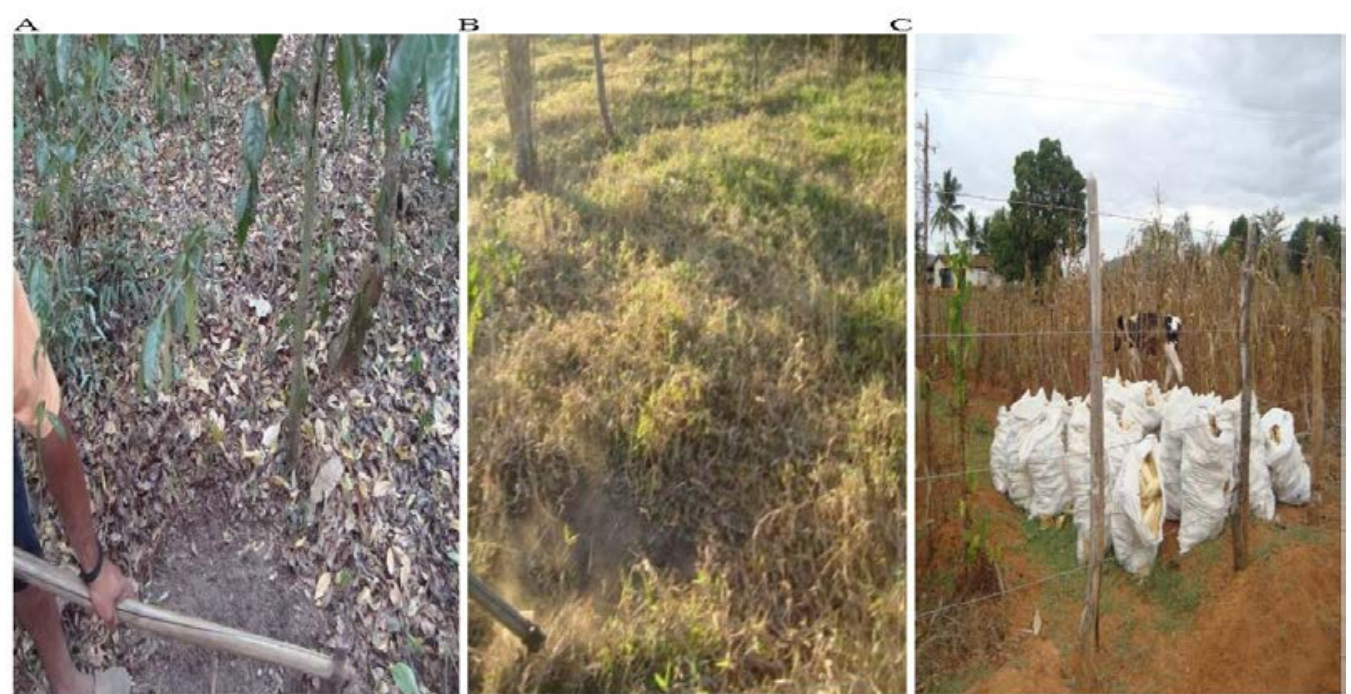

Figura 5. Vegetações no bioma Mata Atlântica: A) Matas, B) Pastagens e C) Milharais (Fotos: Felipe, 2012). 
Tabela 2. Índices de sustentabilidade e ranking das propriedades rurais nos dois biomas isolados e acumuladamente.

\begin{tabular}{|c|c|c|c|c|c|c|c|}
\hline \multirow{2}{*}{$\begin{array}{c}\text { Propriedade } \\
\text { CA }\end{array}$} & \multirow{2}{*}{ IS } & \multicolumn{2}{|c|}{ Rank } & \multirow{2}{*}{$\begin{array}{c}\text { Propriedade } \\
\text { MA }\end{array}$} & \multirow{2}{*}{ IS } & \multicolumn{2}{|c|}{ Rank } \\
\hline & & CA & CA+MA & & & MA & $\mathrm{CA}+\mathrm{MA}$ \\
\hline $\mathrm{P} 1$ & 0,36305 & 24 & 44 & P37 & 0,5203 & 11 & 26 \\
\hline P2 & 0,2934 & 28 & 61 & P38 & 0,6882 & 2 & 6 \\
\hline P3 & 0,36305 & 25 & 45 & P39 & 0,54505 & 10 & 23 \\
\hline P4 & 0,2934 & 29 & 62 & P40 & 0,56305 & 9 & 21 \\
\hline P5 & 0,36305 & 26 & 46 & P41 & 0,6832 & 1 & 5 \\
\hline P6 & 0,40605 & 22 & 34 & P42 & 0,682 & 3 & 4 \\
\hline P7 & 0,54205 & 14 & 24 & P43 & 0,4374 & 12 & 28 \\
\hline P8 & 0,7075 & 3 & 3 & P44 & 0,2955 & 30 & 58 \\
\hline P9 & 0,28105 & 35 & 66 & P45 & 0,60705 & 6 & 10 \\
\hline P10 & 0,6635 & 4 & 7 & P46 & 0,2991 & 29 & 57 \\
\hline P11 & 0,7175 & 1 & 1 & P47 & 0,38585 & 18 & 42 \\
\hline P12 & 0,3866 & 23 & 41 & P48 & 0,4094 & 16 & 33 \\
\hline P13 & 0,5825 & 11 & 17 & P49 & 0,35745 & 23 & 51 \\
\hline P14 & 0,28105 & 34 & 67 & P50 & 0,56765 & 8 & 20 \\
\hline P15 & 0,7075 & 2 & 2 & P51 & 0,43295 & 13 & 29 \\
\hline P16 & 0,28105 & 33 & 68 & P52 & 0,41895 & 14 & 31 \\
\hline P17 & 0,5873 & 10 & 15 & P53 & 0,41895 & 15 & 32 \\
\hline P18 & 0,5311 & 15 & 25 & P54 & 0,37185 & 19 & 43 \\
\hline P19 & 0,28105 & 37 & 69 & P55 & 0,2943 & 31 & 59 \\
\hline P20 & 0,4891 & 16 & 27 & P56 & 0,65415 & 4 & 8 \\
\hline P21 & 0,5873 & 9 & 16 & P57 & 0,31785 & 25 & 53 \\
\hline P22 & 0,36305 & 27 & 47 & P58 & 0,2943 & 32 & 60 \\
\hline P23 & 0,5587 & 13 & 22 & P59 & 0,31425 & 26 & 54 \\
\hline P24 & 0,28105 & 36 & 70 & P60 & 0,6306 & 5 & 9 \\
\hline P25 & 0,28105 & 32 & 71 & P61 & 0,2907 & 34 & 64 \\
\hline P26 & 0,5973 & 7 & 13 & P62 & 0,2934 & 33 & 63 \\
\hline P27 & 0,28105 & 31 & 72 & P63 & 0,31185 & 27 & 55 \\
\hline P28 & 0,40605 & 21 & 35 & P64 & 0,31185 & 28 & 56 \\
\hline P29 & 0,5973 & 6 & 12 & P65 & 0,28385 & 35 & 65 \\
\hline P30 & 0,57405 & 12 & 18 & P66 & 0,36305 & 20 & 48 \\
\hline P31 & 0,28105 & 30 & 72 & P67 & 0,36305 & 21 & 49 \\
\hline P32 & 0,40605 & 20 & 36 & P68 & 0,40585 & 17 & 40 \\
\hline P33 & 0,40605 & 19 & 37 & P69 & 0,36305 & 22 & 50 \\
\hline P34 & 0,5976 & 5 & 11 & P70 & 0,3383 & 24 & 52 \\
\hline P35 & 0,5873 & 8 & 14 & P71 & 0,5721 & 7 & 19 \\
\hline P36 & 0,40605 & 18 & 38 & P72 & 0,28105 & 36 & 73 \\
\hline P73 & 0,42005 & 17 & 30 & & & & \\
\hline
\end{tabular}

IS - Índice de Sustentabilidade; CA - Caatinga; MA - Mata Atlântica.

No geral, as propriedades do bioma Caatinga tiveram IS melhores do que as propriedades da Mata Atlântica. Iguais tendências foram observadas em relação aos atributos físico-químicos, mostrando haver uma sintonia entre estas variáveis estudadas. Pode-se observar que das 73 propriedades pesquisadas e das $34 \mathrm{com}$ IS do tipo insustentáveis, 19 ou 55,88\% foram encontradas na Mata Atlântica, enquanto apenas 15 ou 44,12\% fizeram-se presentes na Caatinga.

Considerando-se as 30 melhores propriedades avaliadas, 17 encontraram-se no bioma Caatinga e apenas 13 no bioma Mata Atlântica. Tendência inversa observou-se analisando as 30 piores, encontrando-se 17 propriedades na Mata Atlântica contra apenas 13 na Caatinga. Isto reforça a tese que de fato a Caatinga 
apresentou aptidão agrícola mais favorável do que a Mata Atlântica, independente da atividade agrícola desenvolvida.

Em ambos os casos, observando as respostas dadas nos questionários, constatou-se que as melhores propriedades em IS foram também as melhores em condições técnicas, econômicas e sociais, reforçando a tese de que estes fatores foram realmente importantes na racionalização do uso do solo, ocorrendo o contrário com as piores.

Pode-se verificar ainda na Tabela 3 que os resultados mostraram não haver propriedades do tipo seriamente insustentável (SI), mas por outro lado revelaram não haver também propriedades sustentáveis (S). Este fato sinalizou que nem tudo foi desanimador, nem tão pouco que tudo foi maravilhoso.

Tabela 3. Classificação dos produtores rurais em função da sustentabilidade das atividades agrícolas nos dois biomas.

\begin{tabular}{c|c|c|c|c|c|c|c}
\hline \multirow{2}{*}{ Classificação } & \multirow{2}{*}{ Intervalos } & \multicolumn{2}{|c|}{ CA } & \multicolumn{2}{c|}{ MA } & \multicolumn{2}{c}{ Total } \\
\cline { 3 - 8 } & & $\mathbf{N}^{\mathbf{0}}$ & $\mathbf{\%}$ & $\mathbf{N}^{\mathbf{0}}$ & $\mathbf{\%}$ & $\mathbf{N}^{\mathbf{0}}$ & $\mathbf{\%}$ \\
\hline S & IS $>0,80 ;$ & 0 & 0 & 0 & 0 & 0 & 0 \\
SA & $0,60<\mathrm{IS} \leq 0,80 ;$ & 4 & 10,81 & 6 & 16,67 & 10 & 13,70 \\
SC & $0,40<\mathrm{IS} \leq 0,60 ;$ & 18 & 48,65 & 11 & 30,55 & 29 & 39,72 \\
I & $0,20<\mathrm{IS} \leq 0,40 ;$ & 15 & 40,54 & 19 & 52,78 & 34 & 46,58 \\
SI & IS $\leq 0,20$ & 0 & 0 & 0 & 0 & 0 & 0 \\
\hline Total & - & 37 & 100 & 36 & 100 & 73 & 100 \\
\hline
\end{tabular}

CA-Caatinga; MA-Mata Atlântica; S-Sustentável; SA-Sustentabilidade Ameaçada; SC-Sustentabilidade Comprometida; I-Insustentável; SI-Seriamente Insustentável. Adaptado de Lopes et al. (2009).

Em linhas gerais, as 73 propriedades se enquadraram na faixa intermediária, compreendendo as classificações do tipo sustentabilidade ameaçada (10 ou 13,70\%), sustentabilidade comprometida (29 ou 39,73\%) e insustentável (34 ou 46,57\%), sugerindo que quase a metade se constituiu em motivos de preocupações, visto encontrarem-se em condições degradáveis e, consequentemente, desagradáveis.

A preocupação foi aumentada devido 39,73\% encontrarem-se em processo de degradação, ou seja, com sustentabilidade comprometida merecendo urgentemente de medidas preventivas para não se tornarem insustentáveis.

Das 73 propriedades, evidenciou-se que $13,70 \%$ foram classificadas como sendo de sustentabilidade ameaçada, sinalizando que pequenos descuidos podem levar a degradações indesejáveis.

Analisando os biomas separadamente, verificou-se que na Caatinga a situação foi menos desanimadora, uma vez que os IS revelaram quase $60 \%(10,81 \%$ de $\mathrm{SA}+48,65 \%$ de SC) de possibilidade de se trabalhar a sustentabilidade contra pouco mais de $45 \%$ (16,67\% de SA + 30,55\% de SC) da Mata Atlântica.

\section{Análise fatorial}

$\mathrm{Na}$ Tabela 4, pode-se verificar como os componentes e a comunalidade se comportaram em relação as 12 variáveis estudadas. A análise fatorial revelou que todas as variáveis apresentaram comunalidade superior a 0,5 , o que significa dizer que mais de $50 \%$ da variância de cada variável estiveram associadas aos respectivos pesos atribuídos (Norusis, 1990).

Das variáveis estudadas, mereceram destaques pelos maiores coeficientes dos índices de sustentabilidades alcançados as seguintes: grau de instrução (GI), cuidados após aplicações de defensivos (CAD), experiência com sustentabilidade (ES) e práticas conservacionistas utilizadas (PCU). Todas estas variáveis, por terem uma estreita relação com os aspectos técnicos, 
Tabela 4. Matriz de cargas fatoriais das variáveis transformadas pelo algorítmo varimax nos cinco componentes principais selecionados dos biomas Caatinga e Mata Atlântica, Paraíba.

\begin{tabular}{|c|c|c|c|c|c|c|c|}
\hline \multirow{2}{*}{$\mathbf{N}^{\mathbf{o}}$} & \multirow{2}{*}{ Variáveis } & \multicolumn{5}{|c|}{ Componentes } & \multirow{2}{*}{$C^{*}$} \\
\hline & & $\mathrm{C}_{1}$ & $\mathrm{C}_{2}$ & $\mathrm{C}_{3}$ & $\mathrm{C}_{4}$ & $\mathrm{C}_{5}$ & \\
\hline 1 & GI & 0,539 & 0,337 & 0,478 & 0,310 & $-0,123$ & 0,744 \\
\hline 2 & PCU & 0,858 & 0,170 & $-0,138$ & 0,137 & $-0,192$ & 0,839 \\
\hline 3 & OS & 0,846 & $-0,015$ & 0,054 & $-0,169$ & $-0,074$ & 0,753 \\
\hline 4 & EAS & 0,591 & $-0,261$ & 0,162 & 0,316 & 0,505 & 0,798 \\
\hline 5 & CAD & 0,370 & 0,853 & $-0,022$ & 0,021 & 0,169 & 0,895 \\
\hline 6 & DEV & $-0,356$ & 0,767 & 0,242 & 0,154 & $-0,191$ & 0,835 \\
\hline 7 & DVD & 0,312 & 0,544 & $-0,430$ & 0,113 & $-0,507$ & 0,848 \\
\hline 8 & EPI & $-0,075$ & 0,231 & 0,877 & $-0,240$ & $-0,196$ & 0,924 \\
\hline 9 & AI & 0,107 & $-0,203$ & 0,702 & 0,447 & $-0,001$ & 0,745 \\
\hline 10 & CUD & 0,018 & 0,107 & 0,050 & 0,887 & $-0,093$ & 0,809 \\
\hline 11 & DLD & $-0,152$ & $-0,035$ & $-0,144$ & $-0,155$ & 0,776 & 0,671 \\
\hline 12 & DES & $-0,028$ & 0,229 & $-0,268$ & 0,476 & 0,533 & 0,636 \\
\hline & $\sum \mathrm{X}$ & 3,028 & 2,726 & 1,562 & 2,296 & 0,608 & \\
\hline & $\mathrm{F}$ & 2,493 & 1,984 & 1,877 & 1,575 & 1,568 & \\
\hline & ância (\%) & 20,775 & 16,533 & 15,642 & 13,125 & 13,066 & \\
\hline & $\begin{array}{l}\text { ia acumulada } \\
\text { (\%) }\end{array}$ & 20,775 & 37,308 & 52,950 & 66,075 & 79,141 & \\
\hline
\end{tabular}

GI-Grau de Instrução; PCU-Práticas Conservacionistas Utilizadas; OS-Opinião sobre Sustentabilidade; EAS-Experiência com Agricultura Sustentável; CAD-Cuidados após Aplicação de Defensivos; DEVDestino das Embalagens Vazias; DVD-Destino da Vegetação Desmatada; EPI-Equipamento de Proteção Individual; AA-Aramazenamento dos Insumos; CUD-Coeficiente de Uniformidade de Distribuição; DLD-Destino do Lixo Domiciliar; DES-Destino do Esgoto Sanitário; *C-Comunalidade; $\sum \mathrm{X}$-Somatórios das componentes; F-Autovalor (raíz característica).

confirmaram o inter-relacionamento entre estes fatores, corroborando o observado por outros autores (Branco, 2003; Lopes et al., 2009).

\section{Agrupamento das propriedades}

Para o agrupamento das áreas envolvendo os dois biomas e três atividades agrícolas em função dos aspectos técnicos, econômicos e sociais definidos pelos questionários aplicados, foram verificadas diferenças significativas confirmadas pelos valores das respostas obtidas. Quatro grandes grupos se formaram: o grupo 1 , com as propriedades P2, P4, P3, P5, P9, $\mathrm{P} 14, \mathrm{P} 16, \mathrm{P} 19, \mathrm{P} 1, \mathrm{P} 20, \mathrm{P} 22, \mathrm{P} 24, \mathrm{P} 25$, P27, P6, P28, P32, P33, P36, P48, P51, P52, P53, P55, P58, P62, P63, P64, P66, P67, P31，P72，P69，P12，P37，P47，P46, P59, P18, P39, P61, P70, P65, P73, P54, P23, P43, P10, P68, P57, P7 e P44; o grupo 2, com as propriedades $\mathrm{P} 8, \mathrm{P} 15, \mathrm{P} 11, \mathrm{P} 30$, P34 e P13; o grupo 3, com as propriedades P17, P21, P26, P29, P35, P56, P60, P71,
P38; P41, P42, P45, P40 e P50; e o grupo 4, com a propriedade $\mathrm{P} 49$.

Pode-se observar na Figura 6 que no grupo 1 foram identificadas as propriedades com sustentabilidade comprometida e/ou insustentáveis, constituindo o maior agrupamento com 52 do total de 73 avaliadas. No grupo 2, em 6 propriedades identificaram-se metade classificada como de sustentabilidade ameaçada e outra metade como comprometida; no grupo 3 com 14, foram encontradas àquelas com sustentabilidade comprometida e/ou ameaçada e no grupo 4 com apenas 1 que foi classificada como insustentável. Verificou-se, portanto, maioria esmagadora das propriedades em condições insustentáveis, o que sugere adoções de ações imediatas no sentido de minimizar tais problemas.

Das 73 propriedades pesquisadas, foram classificadas com base nas respostas obtidas à aplicação dos questionários 10 com sustentabilidade ameaçada, 29 com 


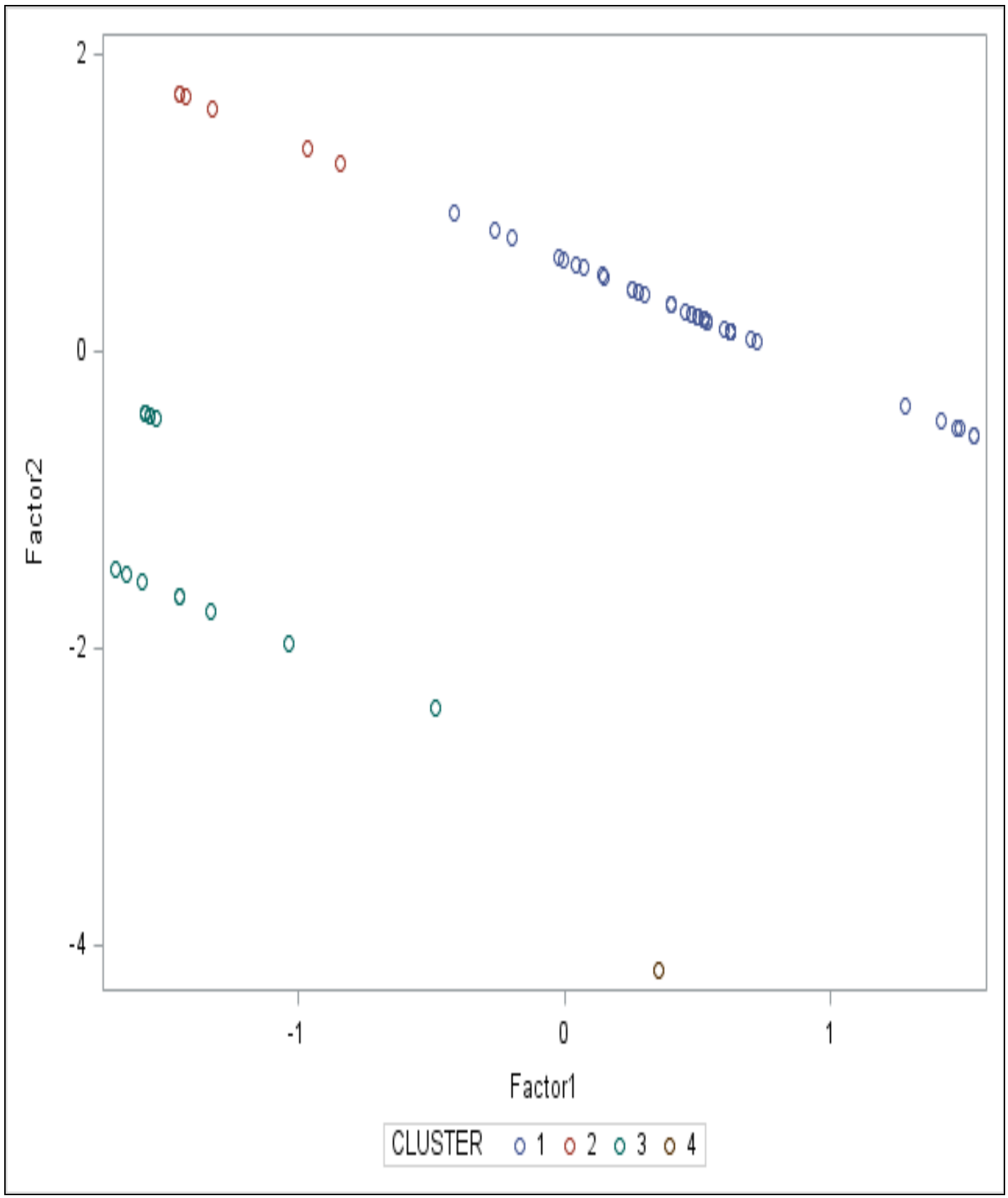

Figura 6. Agrupamento das diferentes áreas de estudo, em diferentes biomas (Caatinga e Mata Atlântica) e atividades agrícolas (Mata em Regeneração, Monocultivo de Pastagem e Monocultivo de Milho), construídos pelos aspectos técnicos, econômicos e sociais identificados através de questionários.

sustentabilidade comprometida e 34 com insustentabilidade. Nenhuma delas foi classificada como seriamente insustentável, mas também nenhuma se enquadrou como sustentável.

Já analisando biomas separadamente, observou-se que das 37 propriedades da Caatinga, 4 foram classificadas com sustentabilidade ameaçada, 18 com sustentabilidade comprometida e 15 com insustentabilidade; enquanto que das 36 propriedades analisadas na Mata Atlântica, 6 foram classificadas com sustentabilidade ameaçada, 11 com sustentabilidade comprometida e 19 com insustentabilidade.

Tanto no geral como isoladamente, apesar da não existência de nenhuma propriedade em situação seriamente 
insustentável, verificou-se ser preocupante o número de propriedades insustentáveis e mais ainda o fato de não existir nenhuma sustentável, indicando a necessidade de providências imediatas no sentido de atenuar estes problemas relacionados ao comprometimento ambiental dos dois biomas.

\section{Conclusões}

1. As condições técnicas, econômicas e sociais influenciaram nos índices de sustentabilidades (IS) das propriedades rurais, revelando que quando estes aspectos foram melhores, maiores foram os IS.

2. A Caatinga foi superior à Mata Atlântica no referente aos IS, não significando por esta razão estar numa condição extremamente favorável em relação à sustentabilidade ambiental, pois também precisa de cuidados e medidas preventivas para racionalizar o uso do solo.

3. Das 73 propriedades avaliadas, às insustentáveis totalizando 34 (46,58\%) encontraram-se em maior quantidade e percentual na Mata Atlântica. Do total das 73 propriedades, 39 ou 53,42\% merecem maiores cuidados para alcançar a sustentabilidade.

4. Nenhuma das 73 propriedades pesquisadas foi plenamente enquadrada como sustentável (IS $>0,80$ ), nem também como seriamente insustentável (IS $\leq 0,20$ ).

5. Os quatro grandes grupos formados de propriedades rurais revelaram que as atividades agrícolas necessitam de gestões imediatas no sentido de minimizar problemas relacionados a sustentabilidade.

6. Com 17 contra 13, a Caatinga superou a Mata Atlântica na análise das 30 melhores propriedades avaliadas; 0 contrário ocorrendo com as 30 piores (17 da Mata Atlântica contra apenas 13 da Caatinga).

7. Para alcançar a sustentabilidade (IS $>0,80)$ os aspectos técnicos, econômicos e sociais precisam ser melhorados nas propriedades rurais em todas as atividades agrícolas desenvolvidas nos dois biomas pesquisados.

\section{Agradecimentos}

Ao Instituto Federal de Rondônia (IFRO) e a Empresa de Assistência Técnica e Extensão Rural de Rondônia (EMATERRO) pela liberação e apoio ao primeiro autor para realização da pesquisa no Estado da Paraíba.

\section{Declaração de conflito de interesses}

Os autores declaram não haver conflitos de interesses.

\section{Referências}

Andrade, J. G. Diagnóstico e intervenção administrativa em fazendas. Lavras: ESAL/FAEPE, 1991.

Barreto, R. C. S.; Khan, A. S.; Lima, P. V. P. S. Sustentabilidade dos assentamentos do Município de Caucaia-CE. Revista de Economia e Sociologia Rural, v. 43, n. 2, p. 225-247, 2005.

Bello, J. L. P. Metodologia científica: manual para elaboração de textos acadêmicos, monografias, dissertações e teses. Rio de Janeiro: UVA, 2008.

Branco, M. C. Avaliação do conhecimento do rótulo dos inseticidas por agricultores em uma área agrícola do Distrito Federal. Revista Horticultura Brasileira, v. 21, n. 3, p. 570573, 2003.

Canuto, J. C. A pesquisa e os desafios da transição agroecológica. Revista Ciência \& Ambiente, v. 27, p. 133-140, 2003.

Carneiro, M. A. C.; Souza, E. D.; Reis, E. F.; Pereira, H. S.; Azevedo, W. C. Atributos físicos, químicos e biológicos de solo de cerrado sob diferentes sistemas de uso e manejo. Revista Brasileira de Ciência do Solo, v. 33, p. 147157, 2009.

Carneiro Neto, J. A.; Andrade, E. M.; Rosa, M. F.; Mota, F. S. B.; Lopes, J. F. B. Índice de Sustentabilidade Agroambiental para o Perímetro Irrigado Ayres de Souza. Revista Ciência Agrotécnica, v. 32, n. 4, p. 1272-1279, 2008.

Fonseca, J. S.; Martins, G. A. Curso de estatística. 6. ed. São Paulo: Atlas, 1996.

Google Earth-Mapas. Disponível em: $<$ http://mapas.google.com>. Acesso em: $08 \mathrm{fev}$. 2014. 
Horn, R.; Herner, F. Avaliação do risco de compactação do subsolo de terras aráveis no nordeste da Alemanha na escala da exploração agrícola. Kiel: Universidade Christian-Albrecht-zu Kiel, Instituto de Nutrição Vegetal e Ciência do Solo, 2008.

Lopes, F. B.; Andrade, E. M.; Aquino, D. N., Lopes, J. F. B. Proposta de um índice de sustentabilidade do Perímetro Irrigado Baixo Acaraú, Ceará, Brasil. Revista Ciência Agronômica, v. 40, n. 2, p. 185-193, 2009.

Masera, O.; Astier, M.; Ridaura, S. L. Sustentabilidad y manejo de recursos naturales: el marco de evaluación (MESMIS). Mexico: Mundi-Prensa, 1999.

Melo, A. S. S. A. Estimação de um Índice de Agricultura Sustentável: o caso da área irrigada do Vale do Submédio São Francisco. Recife: Universidade Federal de Pernambuco, 1999. (Tese de Doutorado em Economia).

Norusis, M. J. SPSS Base System User's Guide. Chicago: SPSS Inc, 1990.
Palácio, H. A. Q. Índice de qualidade das águas na parte baixa da Bacia Hidrográfica do Rio Trussu, Ceará. Fortaleza: Universidade Federal do Ceará, 2004. (Dissertação de Mestrado em Irrigação e Drenagem).

SAS Institute. SAS/STAT 9.3 User's Guide. Cary/NC, USA: SAS Institute, 2011.

Souza, G. H. F.; Brito, R. L.; Dantas Neto, J.; Soares, J. M.; Nascimento, T. Desempenho do Distrito de Irrigação Senador Nilo Coelho. Revista Brasileira de Engenharia Agrícola e Ambiental, v. 5, n. 2, p. 204-209, 2001.

Toledo, L. G.; Nicolella, G. Índice de qualidade de água em microbacia sob uso agrícola e urbano. Scientia Agrícola, v. 59, n. 1, p. 181186, 2002.

Vasconcelos, R. R.; Torres Filho, W. Impactos ambientais das atividades humanas sobre a base de recursos renováveis no semi-árido: relatório preliminar. Brasília: IPEA/SEPLAN, 1994.

Informação da Licença: Este é um artigo Open Access distribuído sob os termos da Licença Creative Commons Attribution, que permite uso irrestrito, distribuição e reprodução em qualquer meio, desde que a obra original seja devidamente citada. 